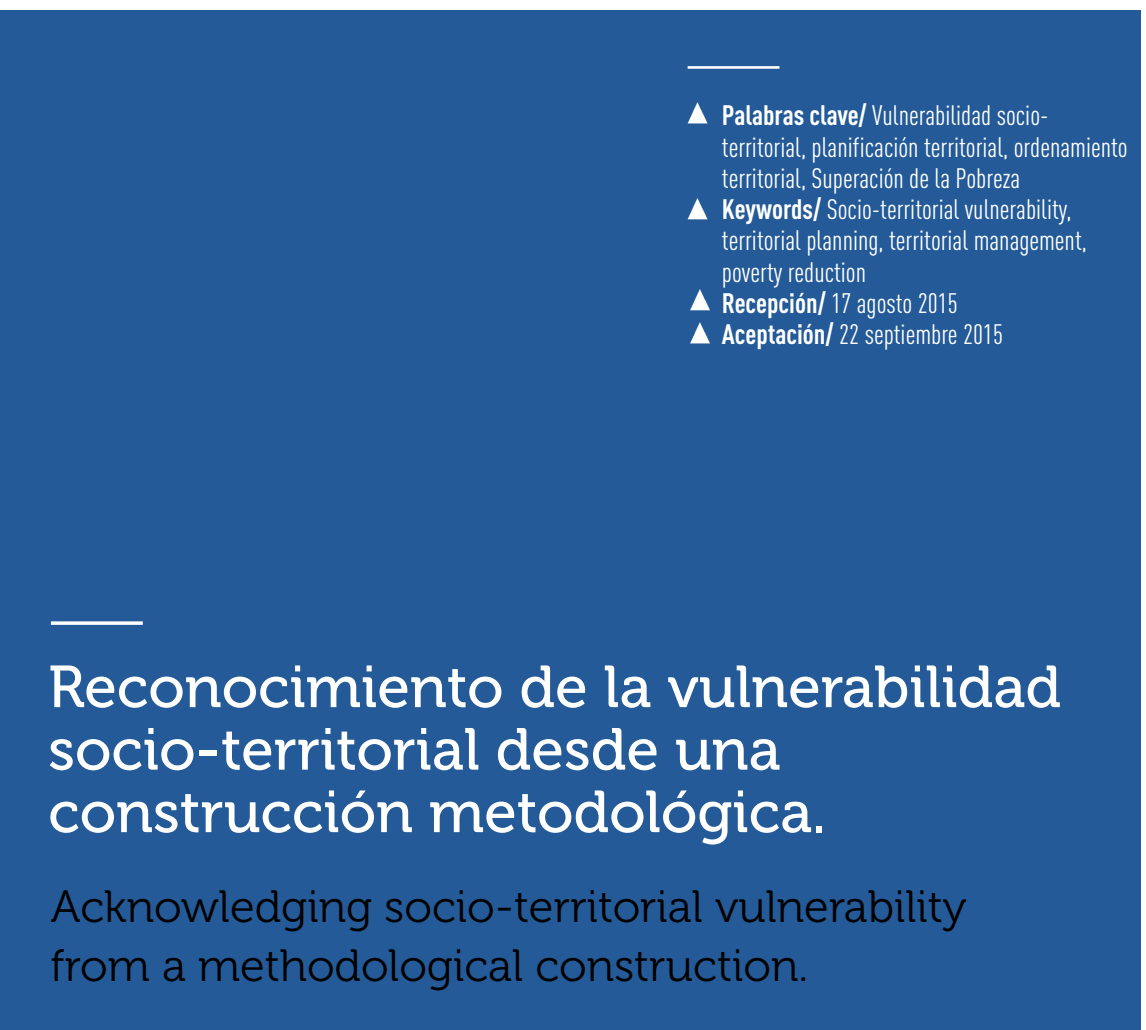

\title{
Verónica Yáñez-Romo
}

Ingeniera Civil en Geografía, Universidad de Santiago, Chile.

Magíster en Planificación y Ciencia

Regional, Instituto Tecnológico de

Karlsruhe, Alemania.

Académico Facultad de Ingeniería

Universidad de Santiago, Chile.

veronica.yanez.r@usach.cl

\section{Carlos Muñoz-Parra}

Arquitecto, Pontificia Universidad Católica de Chile.

Doctor Arquitecto, Universidad Politécnica de Madrid, España

Académico Escuela de Arquitectura,

Universidad de Santiago, Chile.

carlos.munoz.p@usach.cl

\section{Matías Dziekonski-Rüchardt \\ Arquitecto, Universidad de Chile \\ Magíster en Educación, Universidad de Santiago, Chile. \\ Académico, Escuela de Arquitectura, \\ Universidad de Santiago, Chile. \\ matias.dziekonski@usach.cl}

RESUMEN/ En conjunto con la Fundación Superación de la Pobreza y en agradecimiento al proyecto de Investigación DICYT Nº 091312YR, Vicerrectoría de Investigación, Desarrollo e Innovación de la Universidad de Santiago de Chile (USACH), se trabajó sobre el concepto de Vulnerabilidad Social, relacionado estrechamente con los territorios urbanos y las problemáticas que estos presentan. Una vez definido el concepto de Vulnerabilidad Social Territorial, se zonifica el territorio en macro-zonas de estudio, según su división político administrativa, con el fin de identificar factores y variables determinantes que servirán de insumo para generar una propuesta metodológica que permita diagnosticar e identificar los diferentes niveles de vulnerabilidad social a escala regional y comunal, considerando sus efectos territoriales. Esto ayudaría, de forma más eficiente, a las metodologías actuales para la focalización y mejora de políticas, planes y/o programas del gobierno que van dirigidos a Los sectores de mayor rezago y escasos recursos. ABSTRACT/ Jointly with the Fundación Superación de la Pobreza and to express gratitude to DICYT Research Project No. 091312YR, Vice-Rector's Office of Research, Development and Innovation of Universidad de Santiago de Chile (USACH), this work revolves around the concept of social vulnerability, closely related to urban territories and the issues they pose. Once the concept of Territorial Social Vulnerability has been defined, the territory is zoned in macro-areas of study, according to their political-administrative division, in order to identify determinant factors and variables that will serve as input to develop a methodological proposal to diagnose and identify the different levels of social vulnerability at regional and communal scale, considering its territorial impacts. This is expected to efficiently support current government targeting tools and improve policies, plans and/or programs aimed at disadvantaged population groups.

\section{INTRODUCCIÓN. En Chile, las}

actuales políticas públicas del ámbito social dependen no solo del rol del Estado, sino también de una sociedad civil más empoderada y de un mercado versátil y globalizado. Esto ha provocado un conjunto - de visiones, intereses y experiencias ư sectoriales que, con distinta intensidad y - focalización, están reconfigurando cómo la sociedad chilena promueve el desarrollo social y orienta su acción hacia la superación de la pobreza (Comisión Económica para América Latina y el Caribe o CEPAL 1997; PNUD 2000). En este sentido, se observa que existe una carencia de políticas sociales efectivas que den respuesta a las actuales demandas de la sociedad.

Por otro lado, la efectividad e impacto de los programas sociales están por debajo de su potencialidad, y la inserción de criterios y recursos privados en el diseño de planes y programas aún no responde a una acción coordinada, generando un escenario autorreferente y fragmentado desde la perspectiva de los grupos sociales en situación de pobreza (Gacitúa, Sojo y Shelton 2000). Un claro ejemplo de ello, en relación a las políticas de vivienda y territorio, es el mismo Ministerio de Vivienda y Urbanismo (MINVU 2006), que declara que: El Estado, ha generado diversas políticas y líneas de intervención para enfrentar el problema de la pobreza, siendo la producción de viviendas y determinados equipamientos lo más significativo en lo urbano, en consideración al alto déficit heredado. No obstante, lo que ha caracterizado la intervención estatal es que ha operado fundamentalmente desde lo sectorial con escaso énfasis en coordinar y focalizar políticas con una lógica territorial. Las actuaciones sectoriales solo han conseguido aliviar algunas dimensiones del problema de la pobreza (vivienda social, empleo de emergencia, subsidios, etc.), pero no han logrado configurar oportunidades 
sustentables (figura 1). A modo de configurar el perfil de la temática del Hábitat Vulnerable, señalaremos que constituye una forma de intervención humana en el contexto territorial, donde se adecuan espacios de manera directa, paulatina y fundada en visiones de organización política, económica y cultural (Caro 2003) Esto se refiere al afán humano de atender sus requerimientos cotidianos básicos de vida (ser, estar, tener) y sus interrelaciones con sus semejantes y es factible dentro de ciertos parámetros de posibilidades otorgados por el contexto territorial. Es aquí entonces -cuando se toca el aspecto de 'lo posible'- donde surge el tema de las restricciones. Esto se debe a la diversidad de sus componentes y se considera la administración de la gestión de los recursos disponibles, incluyendo la innovación como adaptación en función de la conservación (Vignolo 2004). La clave para adaptarse al cambio del entorno, manteniendo o incrementando el bienestar, está en lo que se conserva, no en lo que se innova. Entonces, es necesario tener una aproximación para saber qué se requiere conservar y qué se precisa innovar, dentro del proceso de generación de un hábitat pertinente, con las necesidades y aspiraciones de un demandante específico, que proviene de distintos contextos territoriales en los cuales éstos se insertan, donde adquieren sus particularidades morfológicas, funcionales y simbólicas. Tienen, además, una amplia diversidad en sus componentes, tanto en su carácter físico-espacial, como climatológico, geográfico y topográfico, los que marcan su carácter sociocultural, sentimientos de identidad, de arraigo, de vínculos sociales y rescate de sus valores culturales.

De esta manera, utilizamos la noción de Vulnerabilidad Social Territorial a partir de lo fundamental que resulta instalar una perspectiva territorial en las decisiones, con la finalidad de superar la parcialidad sectorial, producir cambios en la situación de vulnerabilidad de la población y una integración territorial y sectorial efectiva (Muñoz 1986). Además, entendemos - para efectos de esta reflexión- que la Vulnerabilidad Social Territorial es equivalente a hablar de hábitat vulnerable, toda vez que entendemos al hábitat como una dimensión que contiene implícitamente lo geográfico-territorial y lo social (imagen 1) El Gobierno de Chile desea fortalecer la articulación del sistema de protección social a nivel regional y local. Para ello es necesario identificar de forma integral la localización de los pobladores social, ambiental y económicamente más vulnerables, considerando los efectos propios de cada territorio sobre cada uno de estos (Ministerio de Desarrollo Social 2012). Para dar respuesta a esta necesidad, el Observatorio Social, perteneciente al Ministerio de Desarrollo Social, solicitó al Departamento de Ingeniería Geográfica de la Universidad de Santiago orientación académica para generar una definición propia con respecto al término de Vulnerabilidad Social Territorial y, además, proponer una nueva metodología que permitiese integrar la variable territorial con sus diversas unidades o ámbitos geográficos a escala nacional, regional y comunal. Esto dio motivo al desarrollo de un proyecto de investigación cuyo propósito fue realizar un estudio integrador, considerando el ámbito científico y las ciencias sociales, que sería la base para una propuesta metodológica inspirada en elementos cuantitativos y cualitativos. En este sentido, este trabajo se centra en el estudio de la vulnerabilidad social incorporando la variable territorial e integrando los aspectos sociales, económicos y ambientales.

VERGONZOSA MEDALLA DE ORO

CHILE ES EL PAÍS DE LA OCDE CON MAYOR DESIGUALDAD DE INGRESO: LOS INGRESOS DEL 10\% MÁS RICO EN CHILE SON 26 VECES MÁS ALTOS QUE LOS DEL $10 \%$ MÁS POBRE

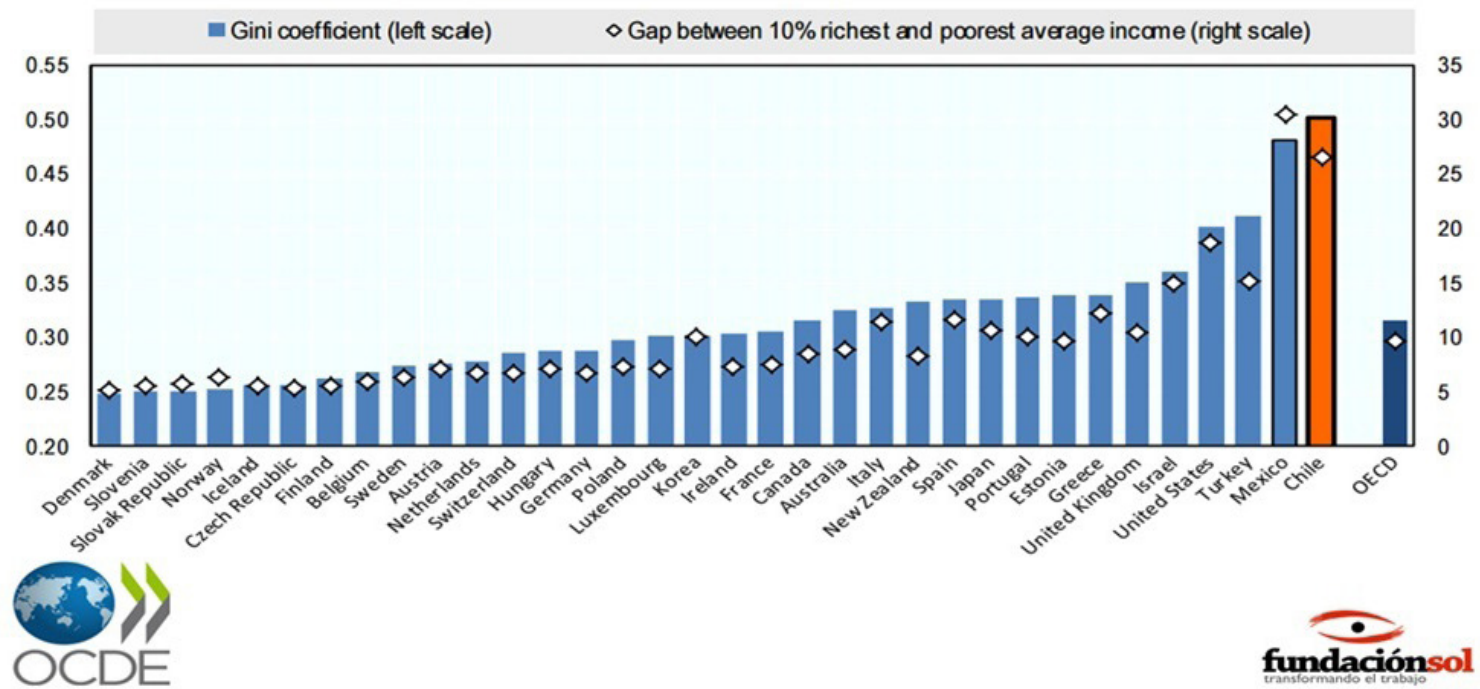

Figura 1. Comparación de la desigualdad en los ingresos. (fuente: Fundación Sol). 


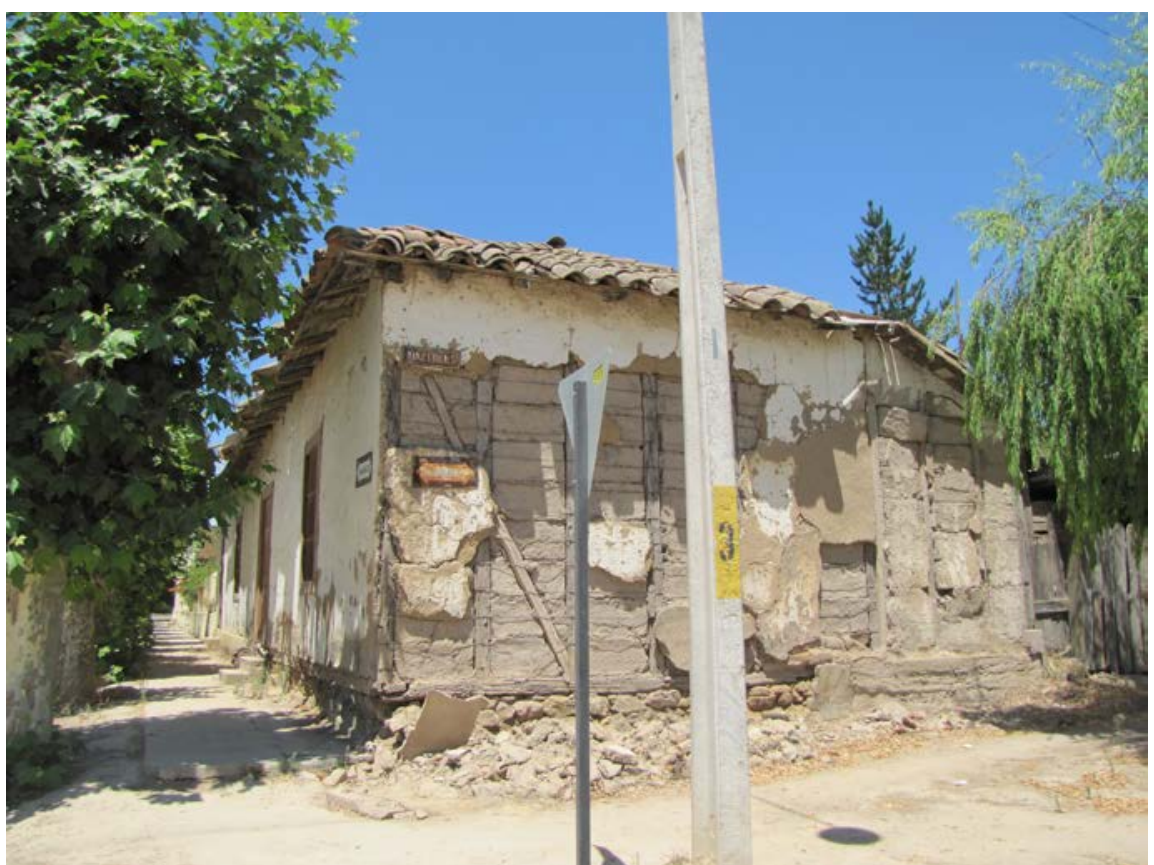

Imagen 1. Vulnerabilidad en los territorios (fuente: Los autores).

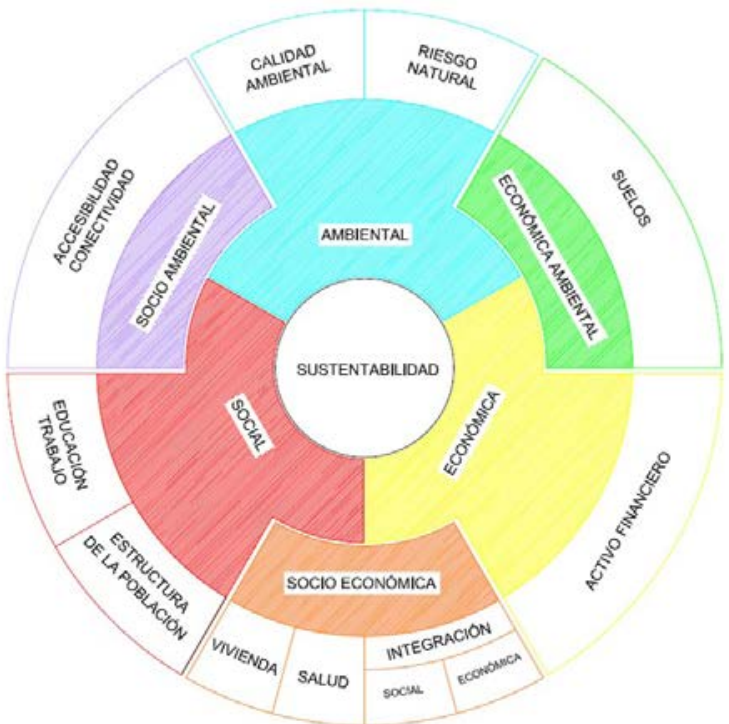

Figura 2. Las Tres Dimensiones y las Sub-Dimensiones de las Variables de Análisis (fuente: Elaboración propia).
2. MARCO TEÓRICO. Etimológicamente el término vulnerabilidad proviene del latín vulnus, que puede traducirse como 'herida' la partícula abilis, que es equivalente a 'que puede'; y, finalmente, el sufijo -dad, que es indicativo de cualidad. De ahí que vulnerabilidad pueda determinarse como "la cualidad que tiene alguien para poder ser herido". El término puede aplicarse a una persona o a un grupo social según su capacidad para prevenir, resistir y sobreponerse de un impacto (Diccionario Esencial de la Lengua Española 2006). Las personas vulnerables son aquellas que, por distintos motivos, no tienen desarrollada esta capacidad y que, por lo tanto, se encuentran en situación de riesgo. El concepto de vulnerabilidad "tiene sus cimientos en las diferencias socioeconómicas que se dan en la sociedad, comprendiendo que se refiere a desventajas o riesgos que la movilización de los activos del que disponen las personas, hogares, comunidades y territorios, no permitan aprovechar oportunidades de integración y movilidad social o contrarrestar riesgos de pérdida de bienestar" (Arriaga 2001). Para Kaztman (2003), "la utilidad de los activos depende de su ajuste a las exigencias de la estructura de oportunidades vigentes". El concepto de vulnerabilidad nace de las ciencias humanas y sociológicas, y fue adoptado y enriquecido por expertos en desarrollo y pobreza. En sus primeras aproximaciones, este término se vincula desde el punto de vista social con situaciones de indefensión, temor e inseguridad que las personas viven, relacionadas a problemas de desocupación bajos ingresos y dificultades en el acceso a servicios básicos. En Europa, a partir de los años 90, este concepto se utiliza en gran parte en la toma de conciencia colectiva de la amenaza que pesa sobre segmentos cada vez más numerosos y mal protegidos de la población. Así, el debate sitúa los términos del problema no en el crecimiento económico ni en la producción de riquezas, sino en la manera de repartirla y de tener acceso a ella. De acuerdo a Naciones 
Unidas-CEPAL (2006) "el dualismo social se plantea a partir de la creciente división social entre los que participan de los beneficios de la modernidad, gozando de ingresos suficientes y estables, y aquellos que excluidos de los beneficios de la modernidad, viven con ingresos insuficientes y trabajan en situación precaria." En Latinoamérica el concepto de vulnerabilidad inicialmente se relaciona a definiciones de vulnerabilidad social desde enfoques de carencia de poder y privación de derechos humanos (Busso 2001); otros se conectan con los conceptos de 'pobreza' y 'niveles socioeconómicos'; y para algunos autores la vulnerabilidad social explica la incapacidad de los grupos más débiles de la sociedad para enfrentar el modelo económico neoliberal vigente (Pizarro 2001) Actualmente, los países latinoamericanos están atravesando intensas transformaciones en el marco de los procesos de globalización, con reestructuración económica que comprende reformas de los Estados, apertura del mercado financiero, incremento de la tercerización, entre otros. Junto a esto, se ha producido un cambio en el tipo de desarrollo social; hay aceptación de la desigualdad y "la noción de igualdad ha sido reemplazada por la de equidad (que es una parte de la igualdad), la agenda social se ha fraccionado y se ha ampliado para contemplar temas como la extrema pobreza, la equidad de género, de raza, de etnia, entre otros" (Kaztman 2003). Es así que en Latinoamérica también se incorpora y estudia el concepto de 'Manejo Social de Riesgo', cuyo enfoque nace en la década del 90 en el Banco Mundial, entendiéndose por riesgo la vulnerabilidad que las personas, las comunidades y los territorios tienen para

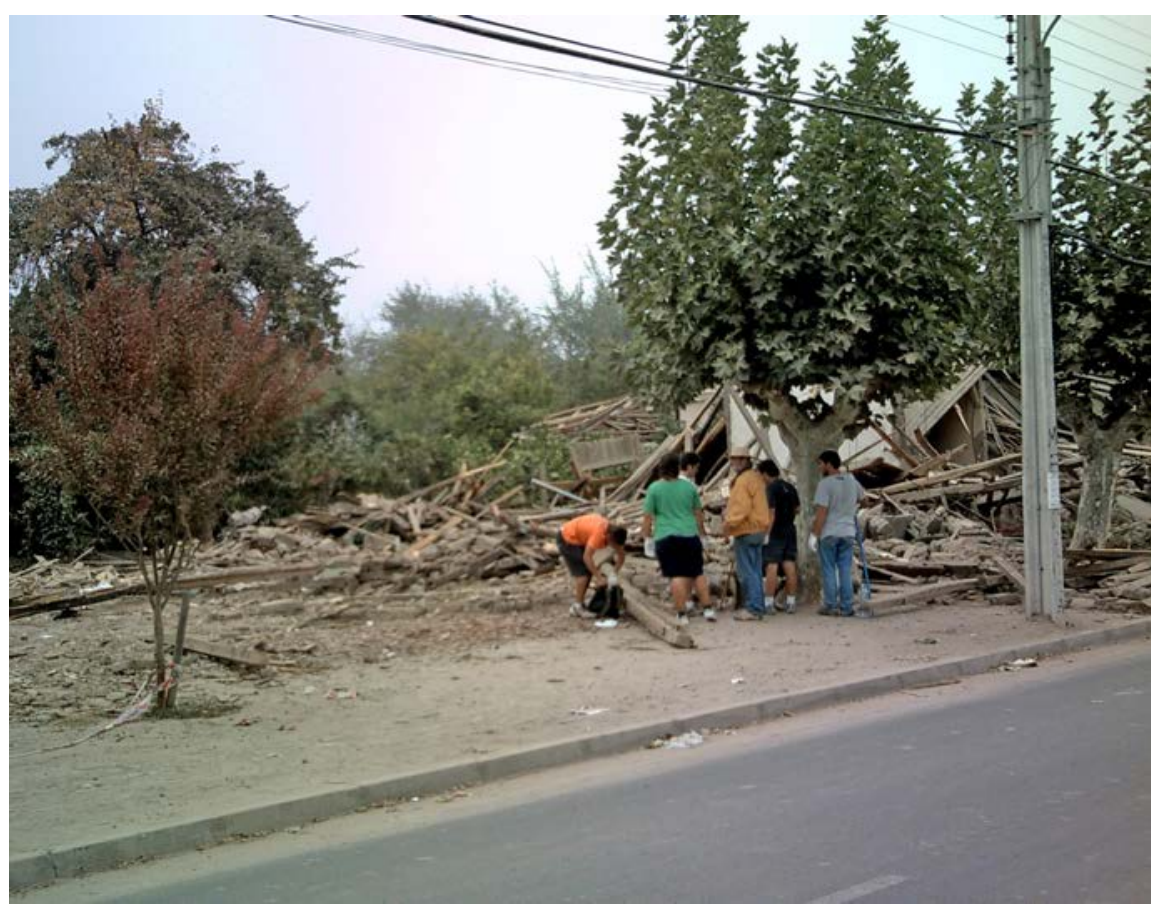

Imagen 2. Optimizar las politicas sociales en el territorio (fuente: Los autores)

enfrentar situaciones que pueden afectarlos negativamente. Este tipo de vulnerabilidad se refiere a la probabilidad de resultar perjudicados por la ocurrencia de sucesos inesperados provocados por los desastres naturales, ambientales o ecológicos. Es así que, en este contexto, existen autores que definen la vulnerabilidad "como la probabilidad de que debido a la intensidad de un evento externo y a la fragilidad de los elementos expuestos ocurran daños en la economía, la vida humana y el ambiente" (Zapata 2000 en CEPAL 2001).

En el caso del Estado chileno, se ha tratado el concepto de vulnerabilidad bajo la perspectiva socioeconómica, desarrollando la 'Estrategia de Protección Social'. Esta se orienta principalmente al bienestar de las personas, garantizando derechos que permiten llevar una vida digna e integrada en el entorno social, cultural y económico. Esto implica un fuerte énfasis preventivo e identificativo de lugares donde la población puede o está en situaciones de carencia de todo tipo que impidan su bienestar, teniendo como desafío fortalecer la articulación del
Sistema de Protección Social' en los niveles regionales y locales. Además, se incorpora explícitamente al 'territorio' como elemento estratégico clave para el diseño y ejecución de las políticas sociales, en el entendido de que es allí donde se desarrolla la vida cotidiana de las personas (Ministerio de Desarrollo Social o MIDEPLAN y Sociedad Alemana de Cooperación Técnica o GTZ 2008).

Esto hace necesario recopilar y revisar las definiciones sobre el concepto de Vulnerabilidad Social Territorial, para escoger o crear una propia, que permita generar una nueva herramienta metodológica integral que haga posible la identificación y caracterización de los tipos de vulnerabilidad (social, económica, ambiental) que pueden estar presentes en un determinado territorio. Lo anterior con el fin de mejorar la localización de los pobladores y su calidad de vida, y el impacto de las políticas sociales al evaluar y controlar los programas sociales ya existentes por parte del Ministerio de Desarrollo Social en Chile. 


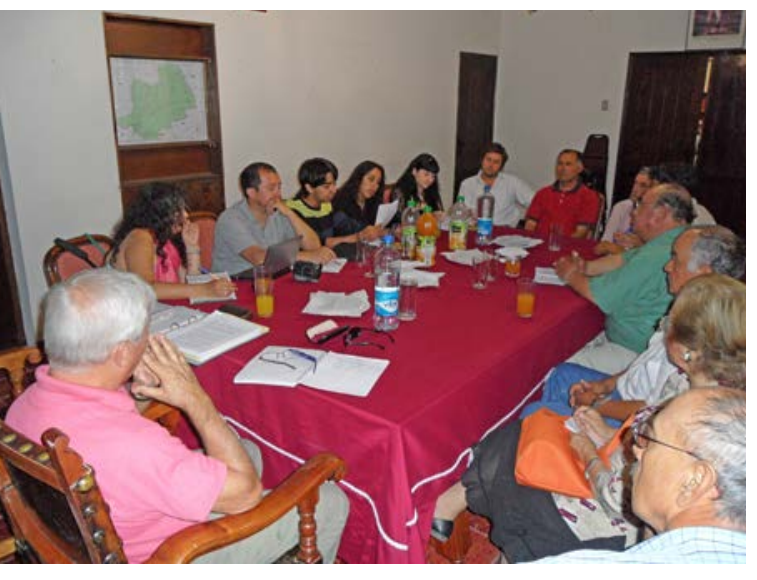

Imagen 3. Validación de decisiones (fuente: Los autores).

2.1. CONCEPTO DE VULNERABILIDAD SOCIAL TERRITORIAL. Para

la realización del diagnóstico y la

determinación del instrumento que permita

identificar los niveles de vulnerabilidad

social-territorial de la región, se realizó

una revisión bibliográfica de los diferentes

conceptos y definiciones que abarcan este

término. Producto de ello se adoptó la definición de Vulnerabilidad Social Territoria

utilizada por el Ministerio de Desarrollo

Social (MDS, ex MIDEPLAN) y la Agencia

Alemana de Cooperación Técnica (GTZ)

surgiendo la definición que aquí se presenta

de la articulación de ambas instituciones.

De acuerdo a estas instituciones, se

entiende la Vulnerabilidad Social Territorial

como "la incapacidad de impedir que acontecimientos de diversa índole afecten negativamente las condiciones de vida de la población que habita un territorio determinado, sea por falta o insuficiencia de activos protectores de riesgos como por la falta de condiciones para aprovechar el flujo

\section{de oportunidades."}

\subsection{DEFINICIÓN DE DIMENSIONES,} SUBDIMENSIONES Y VARIABLES.

A partir de esta definición, se identificó un conjunto de cincuenta y un variables considerando tópicos ambientales, sociales y económicos. Se definieron, luego de una exhaustiva revisión bibliográfica y de un proceso de validación en varias sucesivas mesas de trabajo con expertos para finalmente agruparlas en once subdimensiones que se definían a partir de seis dimensiones, que son las que caracterizan el análisis de procesos sustentables y de una intersección entre ellas. El resultado -excluyendo- las variables es como sigue: Como se puede apreciar en figura 2 , de las tres dimensiones fundamentales constitutivas del concepto de sustentabilidad -ambiental, social y económica- se derivan dos tipos de subdimensiones. Tenemos aquellas propias de las tres dimensiones originales, que son cinco (Calidad Ambiental, Riesgo Natural, Activo Financiero, EducaciónTrabajo y Estructura de la Población) y las que se originan de una intersección entre estas, que son seis (AccesibilidadConectividad, Vivienda, Salud, Integración Social, Integración Socio-Económica y Suelos). A continuación, se definen las seis dimensiones y las once sub-dimensiones que componen el modelo propuesto y se bosquejan las cincuenta y un variables que surgen de las sub-dimensiones (que no solo las constituyen, sino que terminan por definirlas).

Dimensión Ambiental. Constituida por dos sub-dimensiones.

- Sub-Dimensión Calidad Ambiental.

Se refiere al estado y disponibilidad de recursos tales como el agua, el aire, el suelo, el porcentaje de superficies protegidas y la biodiversidad existente.

- Sub-Dimensión Riesgo Natural. Se refiere a fenómenos relativos a la inestabilidad geológica y vulnerabilidad frente a maremotos

Dimensión Socio-Ambiental. Emerge como una dimensión de solo un componente, correspondiente a la articulación de dos de las dimensiones principales: dimensión social y dimensión ambiental.

- Sub-Dimensión Accesibilidad-

Conectividad. Se refiere al acceso y disponibilidad a servicios en sus diferentes niveles, tales como transporte, salud, educación, cultura, servicios bancarios, telecomunicación, agua y energía eléctrica. Dimensión Social. Constituida por dos subdimensiones: educación y trabajo.

- Sub-Dimensión Educación. Características de las condiciones educacionales que posee la población en un territorio dado, tales como escolaridad promedio, tasa de alfabetización y resultados de pruebas estandarizadas como el SIMCE (Sistema de Medición de la Calidad de la Educación) u otras

- Sub-Dimensión Trabajo. Características de las condiciones ocupacionales que posee la población de un territorio en particular, tales como la empleabilidad juvenil y los ingresos del trabajo en el hogar.
Dimensión Socio-Económica. Emerge como una dimensión de cuatro componentes, que corresponden a la articulación de dos de las dimensiones principales: dimensión social y dimensión económica.

- Sub-Dimensión Hábitat. Dice relación con las condiciones físicas de la vivienda y su entorno, tales como el número de viviendas no recuperables, la disponibilidad de agua potable y de alcantarillado de aguas servidas, tipos de materialidad de la vivienda, niveles de hacinamiento, disponibilidad de espacios públicos para el esparcimiento y de infraestructura comunitaria y su uso.

- Sub-Dimensión Salud. Relacionada con las características de atención e infraestructura que poseen los servicios de salud, como también con las condiciones de salud que posee la población (indicadores nutricionales, dotación de personal por establecimiento, tipo de recintos de salud y complejidad de enfermedades que presentan)

- Sub-Dimensión Integración Social.

Proceso donde personas excluidas participan en agrupaciones ciudadanas de un determinado territorio. Se expresa en la asociatividad, la actividad en las organizaciones comunitarias, la participación en los gobiernos locales y regionales, las inversiones del sector privado para mejorar el bienestar de la población y la identificación de los habitantes con su entorno.

- Sub-Dimensión Integración

Socioeconómica. Características socioeconómicas de la población en un territorio. Se expresa en la tasa de detenciones, percepción de seguridad, línea de pobreza y línea de indigencia.

Dimensión Económica. Está constituida por una sub-dimensión.

\section{- Sub-Dimensión Activo Financiero:}

Disponibilidad y procedencia de recursos económicos que poseen las autoridades territoriales.

Dimensión Económico-Ambiental. Emerge como dimensión de un componente que corresponde a la articulación de dos de las dimensiones principales: dimensión ambiental y dimensión económica.

- Sub-Dimensión Suelos: Tiene relación con las condiciones de uso de suelo para fines antrópicos (valor promedio del terreno - $m^{2}$ o Há-) y al potencial del mismo como recurso para desarrollar diferentes actividades. 
3. CONCLUSIÓN. El modelo propuesto pretende aportar una metodología de análisis territorial enfocada en la vulnerabilidad socio-territorial, entregando una herramienta eficiente para la toma de decisiones en materia de protección social que permita propender a medidas de mejoramiento, con el fin de optimizar la gestión del desarrollo y de las políticas sociales en las regiones de Chile (imagen 2). El modelo está compuesto por una serie de Variables y Sub-dimensiones, que pueden ser encasilladas en tres dimensiones: Físico-Ambiental, Social y Económica. Estas variables fueron validadas por una serie de mesas de trabajo compuestas por profesionales de distintas áreas, permitiendo transversalidad al momento de elegir el grupo de variables que componen el indicador VST (Vulnerabilidad Social Territorial).

Esta metodología se aplicó en la Región de Antofagasta, pudiéndose determinar -a nivel comunal- cuáles eran, del total de las nueve comunas que la componen, las comunas más vulnerables y cuáles eran las dimensiones, sub-dimensiones y variables más críticas, tanto en general como en cada comuna. Esto la constituye como una interesante herramienta que, complementada con otras, permitiría mejorar los criterios y efectividad para focalizar la inversión en grupos sociales que la requieran, tanto por parte del Estado como por instituciones privadas dispuestas a hacerlo.

A diferencia de los modelos netamente econométricos y/o estadísticos empleados por Ministerio de Desarrollo Social (MDS) y otros organismos del área, esta propuesta considera una exhaustiva caracterización del área de estudio. De esa forma, se indaga la VST y sus parámetros para cada territorio en particular (Cubillos, Mejías y Yáñez 2013) Al estudiar la VST por medio de variables y sub-dimensiones abarcadas en las dimensiones Social, Físico-Ambiental y Económica, el concepto otorga a la representación un carácter integral desde el punto de vista de la disciplina territorial. De igual modo, es preciso conservar dicho carácter en la aplicación de este instrumento, dado que cualquier mal interpretación u omisión de información podría jugar en contra de una adecuada implementación del modelo.

La creación de un set de variables para cada sub-dimensión y dimensión estudiada brindará a quien aplique el instrumento un punto de partida sustentado en un exhaustivo estudio bibliográfico y trabajo con expertos del área. Sin embargo, dicho punto debe ser validado por expertos locales del área de estudio. Finalmente, este proceso otorga al modelo un carácter transversal, que no excluye las particularidades de cada territorio (imagen 3). Por otro lado, involucrar la participación de los estamentos privado, público y sociedad civil en la construcción de parámetros y ponderadores, otorgará al modelo el carácter de representatividad territorial. No obstante, si la muestra no es equitativa entre las partes, dichos ponderadores no serán representativos y, por lo tanto, es un aspecto a cuidar en la aplicación.

$\Delta 0$

\section{REFERENCIAS}

Arriaga, C., 2001 “Servicios Sociales y Vulnerabilidad en América Latina: Conceptos, medición e indagación empirica." En CEPAL, Seminario Internacional Las diferentes expresiones de la vulnerabilidad social en América Latina y el Caribe. Comisión Económica para América Latina y El Caribe, Naciones Unidas, Santiago de Chile, Chile.

Busso, G., 2001. "Vulnerabilidad Social, Nociones e Implicancias de Politicas para Latinoamérica a inicios del siglo XXI." En CEPAL, Seminario Internacional Las diferentes expresiones de la vulnerabilidad social en América Latina y el Caribe. Comisión Económica para América Latina y El Caribe, Naciones Unidas, Santiago de Chile, Chile

Caro, E., 2003. "La Vulnerabilidad Social como enfoque de análisis de la política de asistencia social para la población adulta mayor en México. Simposio Viejos y Viejas Participación, Ciudadanía e Inclusión Social." 51 Congreso Internacional de Americanistas. Santiago

CEPAL. 1997. Panorama Social de América Latina. Santiago.

CEPAL, 2001. "La Vulnerabilidad Social y sus Desafíos: una mirada desde América Latina”. Pizarro R. . Santiago.

Cubillos, J. Mejías, A. y Yáñez, V.. 2013. Propuesta metodológica para identificar la Vulnerabilidad Social Territorial VST. Caso de estudio: Región de Antofagasta. (Trabajo de Titulación) Facultad de Ingeniería, Departamento de Ingeniería Geográfica, Universidad de Santiago de Chile, Santiago, Chile.

Diccionario Esencial de la Lengua Española. 2006. Versión digital. Enlace obtenido en 05 del 09 del 2015. http://www.rae.es/recursos/diccionarios/desen Vulnerabilidad.

Fundación Sol. Gráfico de Comparación de la desigualdad en los ingresos. Recuperado de https://twitter.com/ lafundacionsol/status/602630326322593792

Gacitúa, E. Sojo, C. y Shelton. D. 2000. Exclusión Social y Reducción de La Pobreza en América Latina y e/ Caribe. San José: FLACSO, Banco Mundial. Recuperado de http://info.worldbank.org/etools/docs/ library/44254/ExclusionSocialyReducciondePobrezaEnAL.pdf
Kaztman, R. CEPAL, 2003. "Notas sobre la medición de la Vulnerabilidad Social." Serie Medio Ambientey Desarrollo, 75

Ministerio de Desarrollo Social, MIDEPLAN. 2012. Observatorio Social. Recuperado de http://observatorio. ministeriodesarrollosocial.gob.cl/

Ministerio de Desarrollo Social, MIDEPLAN y Sociedad Alemana de Cooperación Técnica, GTZ, 2008. Vulnerabilidad Social Territorial: Concepto, Indicadores y Gestión Territorial en el marco de/ Sistema de Protección Social.

Ministerio de Vivienda y Urbanismo, MINVU, 2006. Medición del Déficit Habitacional. Recuperado de www. minvu.cl/opensite_20070411164536.aspx

Muñoz, C., 1986. La Problemática Habitacional chilena 1964-1984: Un Análisis de las Posibilidades de la Autoconstrucción en una Futura Politica de Vivienda como Mecanismo Complementario para su Solución (Tesis de Doctorado). Escuela Técnica Superior de Arquitectura, Universidad Politécnica de Madrid, Madrid España.

Naciones Unidas y Comisión Económica para América Latina y el Caribe, CEPAL. 2006. La protección socia de cara al futuro: Acceso, financiamiento y solidaridad. XXXI Periodo de Sesiones, Montevideo, Uruguay Pizarro, R., 2001. La Vulnerabilidad Social y sus Desafios: Una mirada desde América Latina. Santiago: Comisión Económica para América Latina y el Caribe, CEPAL. Recuperado de http://repositorio.cepal.org/ bitstream/handle/11362/4762/1/S0102116 es.pdf

PNUD. 2000. Más Sociedad para Gobernar el Futuro.

Vignolo, C..2004. "Desencadenando la Innovación en la Empresa del Tercer Milenio." En Trend Management, 6. 